\title{
Outcome of children admitted to a general high- care unit in a regional hospital in the Western Cape, South Africa
}

\author{
I Kruger, ${ }^{1}$ FCPaed (SA); R Gie, ${ }^{1}$ FCPaed (SA); J Harvey, ${ }^{2}$ PhD; M Kruger, ${ }^{1}$ MMed Paed, FCPaed (SA), MPhil, PhD \\ ${ }^{1}$ Department of Paediatrics and Child Health, Faculty of Medicine and Health Sciences, Stellenbosch University, Cape Town, South Africa \\ ${ }^{2}$ Centre for Statistical Consultation, Stellenbosch University, Cape Town, South Africa
}

Corresponding author: I Kruger (immie78@hotmail.co.uk)

\begin{abstract}
Background. Critically ill children are often managed in non-tertiary general intensive care units admitting both adults and children, but few data are currently available regarding paediatric outcomes in these general units.

Objective. To determine the outcome of critically ill neonates and children admitted to a general high-care unit in a large regional hospital in the Western Cape, South Africa.

Methods. This was a retrospective descriptive analysis of outcome of all neonatal and paediatric $(<13$ years of age $)$ patients admitted with non-surgical disease, during a 1-year period, to a general high-care unit at a large regional hospital in Worcester, South Africa. Data included demography, admission time, length of stay, diagnoses, HIV status, therapeutic interventions and outcome. The primary outcome was defined as successful discharge, transfer to a central hospital or death.

Results. There were 185 admissions, with the majority $(83 \%)<12$ months of age (median age 3.7 months; range 0 - 151 months) and a male:female ratio of $1.3: 1$. The majority $(70 \%)$ were successfully discharged, while $24 \%$ were transferred to a tertiary paediatric intensive care unit (PICU) and only 6\% died. Causes of death included acute lower respiratory tract infections (33\%), acute gastroenteritis (33\%), birth asphyxia (16\%) and complications of prematurity (16\%). Nasal continuous positive airway pressure $(p<0.001)$, ventilation $(p<0.001)$ and HIV infection $(p=0.010)$ were associated with transfer to a PICU in a central hospital or death.

Conclusion. The majority of children (70\%) requiring admission to a general high-care unit in a regional hospital were successfully treated and discharged. These good outcomes were only achievable with a good transfer system and supportive tertiary healthcare system.
\end{abstract}

S Afr J Child Health 2016;10(3):156-160. DOI:10.7196/SAJCH.2016.v10i3.981

Globally, more than 10 million children die annually, and the majority of these deaths occur in sub-Saharan Africa (41\%) and southern Asia (34\%), where under-5 mortality is attributed mainly to diarrhoea, pneumonia, measles, malaria, HIV/AIDS and malnutrition. ${ }^{[1,2]}$ Neonatal deaths occur due to asphyxia, preterm delivery, sepsis and tetanus. ${ }^{[2]}$ A country's epidemiological profile and health system largely dictate the implementation and development of public health interventions that will improve childhood survival. ${ }^{[1]}$ Little is known about the need for paediatric high care and critical care in low- and middle-income countries, but published results indicate an inadequate quality of care with high mortality rates. ${ }^{[3]}$ Lack of infrastructure, human resources and expert support leads to children being managed in general wards with low nurse-to-patient ratios, resulting in an increased risk of nosocomial infections and death. ${ }^{[3-6]}$

Paediatric intensive care is defined as a 'physical space that is designated to manage critically ill children who require comprehensive and intensive healthcare, and should be available to all children irrespective of where they live. ${ }^{[7]}$ Research in developed countries suggests that paediatric intensive care units (PICUs) should be centralised with good retrieval services to ensure safe access. There is no clear guidance regarding the infrastructure of a PICU in a low- or middle-income country where transport services often are weak or non-existent. ${ }^{[8]}$ Transfer of critically ill children poses a challenge in South Africa (SA), and a study from Cape Town revealed a high incidence of transfer-related adverse events in children transferred from non-academic metropolitan hospitals to tertiary care centres. ${ }^{[9]}$ Critically ill children are, therefore, often managed by non-specialist staff in non-tertiary general intensive care units admitting both adults and children, but few data are currently available regarding paediatric outcomes in these general units. ${ }^{[9]}$ This study was done to determine the outcome of neonates and children admitted to a general high-care unit under the care of paediatricians at a large regional hospital in the Western Cape Province, SA.

\section{Methods}

The study was conducted at the large regional hospital in Worcester, SA. Worcester Hospital is the regional hospital for the Winelands/ Overberg region of the Western Cape, with $\sim 1$ million inhabitants, of whom $1.6 \%$ were $<1$ year of age. The infant mortality and childhood mortality rates at the time of the study were 25.3 and 31.2/1 000 live births, respectively. ${ }^{[10]}$ The hospital has a five-bed general high-care unit. One of these beds is dedicated to paediatric care. The care of neonates and children admitted to the high-care unit takes place under the supervision of a paediatrician, supported by either a paediatric registrar, medical officer or intern depending on the call list. The nursing staff includes six registered nurses trained in adult intensive care and one registered nurse trained in paediatric intensive care. Children and neonates admitted to the general high-care unit can be ventilated for a limited period, and if not weaned within 48 72 hours are transfered to a central PICU. High care is also provided in the neonatal ward but if mechanical ventilation is required, the neonate is transferred to the general high-care unit. There are no other high-care beds in the hospital, therefore all children requiring high or intensive care are admitted to the general high-care unit. This study is a retrospective review of patient records, capturing data for a 12-month period from 1 July 2008 until 30 June 2009. The high-care admissions records were used to determine all neonates and children admitted. The medical record of each patient was retrieved from the 
hospital records and the data recorded on a case-recording form.

Ethical approval was obtained from the Health Research Ethics Committee at Stellenbosch University (N11/07/209). A waiver of individual consent was granted. Consent from the hospital management was obtained to access the patient records.

All consecutive admissions of neonates and children meeting the inclusion criteria were included. Inclusion criteria were: all children younger than 13 years of age admitted with a non-surgical condition. Exclusion criteria were: (i) children older than 13 years of age at admission; (ii) children admitted with a surgical condition; and (iii) children directly transferred to tertiary facilities.

\section{Definitions and outcomes}

The three primary outcome measurements were: death, transfer to a central hospital and successful discharge. Death was defined as a death that occurred during the general highcare unit admission. Transfer to dedicated PICUs in central hospitals was defined as a transfer. Successful discharge was defined as discharge from the general high-care unit to a step-down facility, paediatric inpatient ward or home.

Ventilatory support included nasal continuous positive airway pressure (nCPAP) and/or mechanical ventilation. For the purposes of this study nasal cannula oxygen was not considered an intervention and high-flow nasal oxygen was not used during the study period. Transfusions were defined as infusion of blood, blood products or albumin, while inotropic support referred to intravenous infusion of dopamine, dobutamine, adrenaline or a combination of these drugs. The unit did not have a transfusion policy; transfusions were administered at the clinician's discretion. Neonates and children were weighed on admission. If they were too ill to be weighed, their weights were obtained from the most recent weight in the Road to Health Booklet. Severe malnutrition was defined as a weightfor-age $z$-score (WAZ) of $<-3 \mathrm{SD}$, while moderate malnutrition was defined as a WAZ of between -3 and -2 SD. The WAZ was determined for each patient's admission weight. For children 0 - 5 years, the WHO Anthro computer program (version 3.2.2) was used. For children $>5$ years, the Centre for Health Statistics values were used. To determine the WAZ for premature infants, the Fenton Growth charts were used. ${ }^{[1]}$ These charts reflect actual age instead of completed weeks.

After hours was defined as admission after $17 \mathrm{~h} 00$, before $08 \mathrm{~h} 00$ on weekdays and from Friday 17h00 until Monday 08h00 on weekends. Public holidays were regarded as after hours.

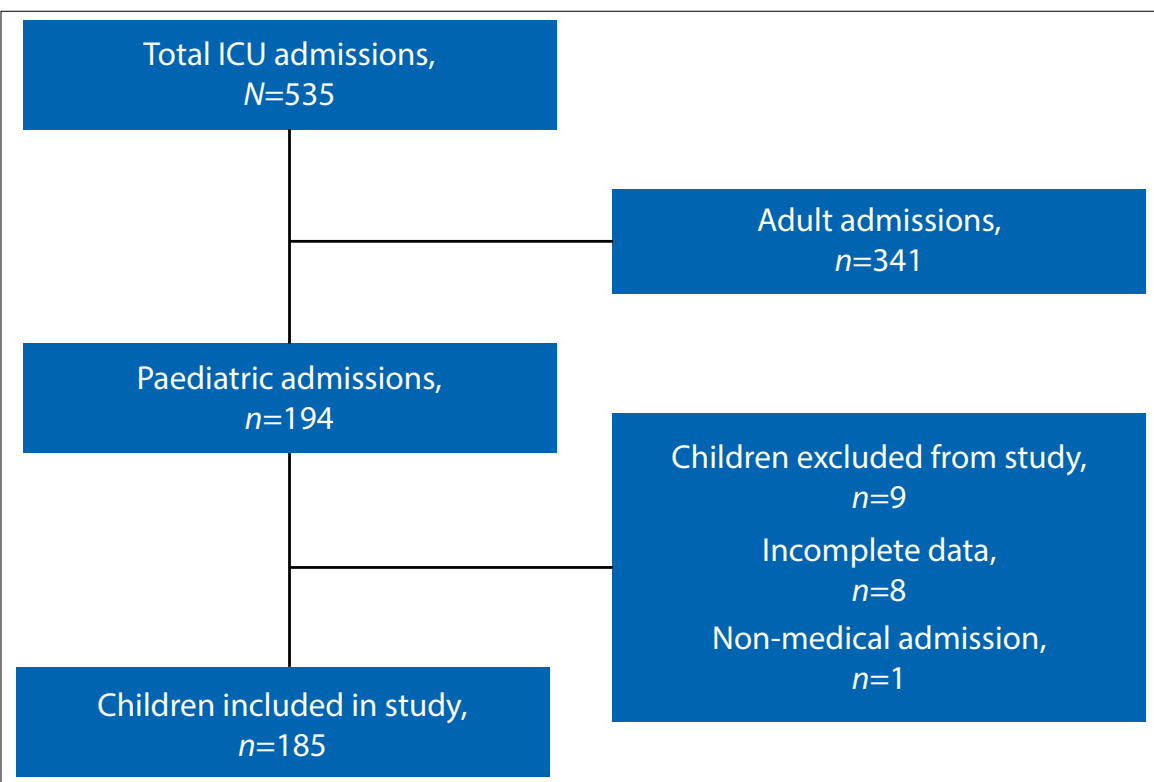

Fig. 1. Sampling strategy.

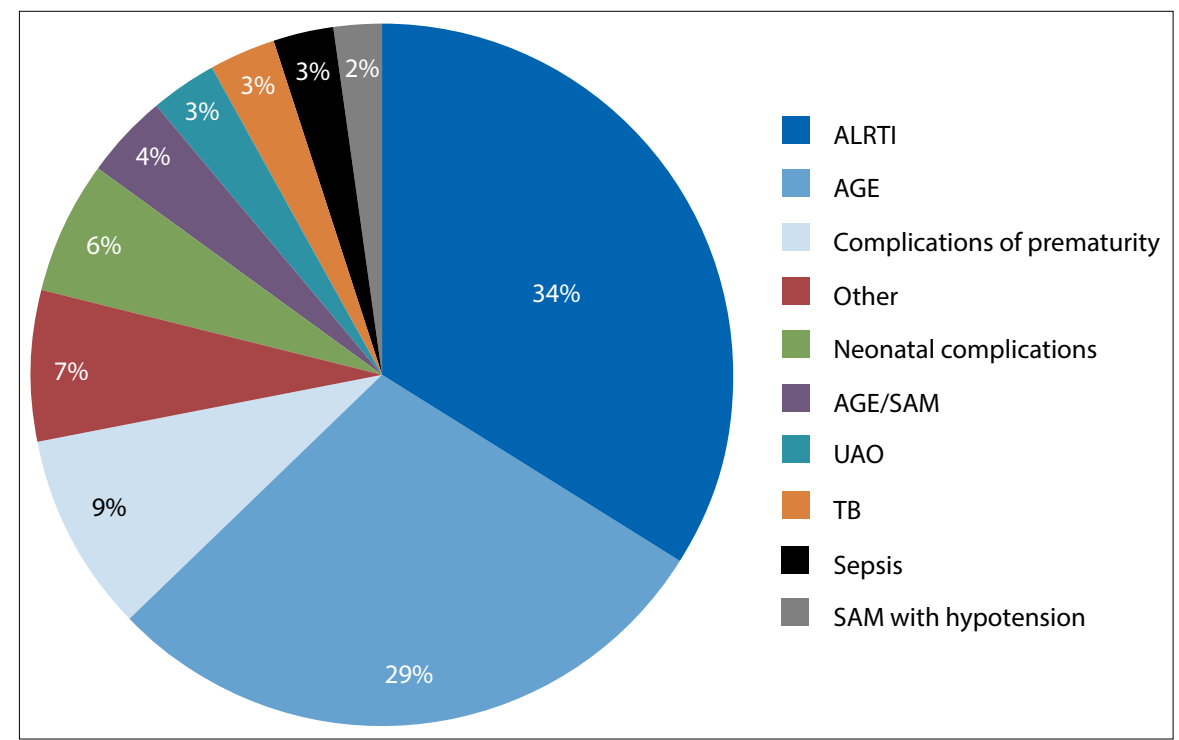

Fig. 2. Reasons for admission to ICU. (SAM = severe acute malnutrition.)

Continuous variables were not normally distributed. The descriptive data are presented as the median with the data range. To compare continuous variables by group ('Successful discharge,' 'Transfer' or 'Died') a Kruskal-Wallis analysis of variance (ANOVA) was used, since data were not normally distributed. In the case of overall significance, post-hoc testing was applied, correcting for multiple comparisons using the Bonferroni approach. Comparisons of nominal variables and group were performed using the $\chi^{2}$ test for association. A significance level of $95 \%$ was applied to all analyses.

\section{Results}

More than a third (36\%, $n=194 / 535)$ of the admissions to the general high-care unit were paediatric admissions, of whom $22 \%$
( $n=41 / 194)$ were neonates. Of the 194 paediatric admissions, 185 (95\%) patient records were analysed. Eight records were excluded owing to incomplete data and one record owing to the patient having a surgical condition (Fig. 1).

The male to female ratio was $1.3: 1$, and the majority (83\%) of the patients were younger than 12 months at the time of admission. The median age was 3.7 (range 0 - 151) months. The median WAZ was -1.54 SD (range $-6.5 \mathrm{SD}-1.88 \mathrm{SD})$, with $25 \%(n=46 / 185)$ of children severely malnourished (WAZ $<-3 \mathrm{SD})$, and $16 \%(n=30 / 185)$ moderately malnourished (WAZ $-2--3 \mathrm{SD}$ ).

Fig. 2 provides a breakdown of the reasons for admission to the general high-care unit. This reflects only the main reason for admission and not underlying comorbidities. 
The majority of admissions were due to acute lower respiratory tract infections (ALRTI) (34\%, $n=63 / 185)$ and acute gastroenteritis (AGE) $(29 \%, n=54 / 185)$.

The majority (53\%) were directly admitted from the casualty unit, $33 \%(n=61 / 185)$ from inpatient wards, $12 \%(n=22 / 185)$ from other referral healthcare facilities surrounding the hospital and 3 children $(<1 \%)$ from theatre. The patients admitted directly from theatre included 2 children intubated in theatre due to upper airways obstruction and 1 infant admitted post caesarean section due to respiratory distress. The majority of admissions (62\%) occured after hours. The median duration of admission was 1.83 days (range 1 hour - 13.4 days). Ventilatory support was provided to $65 \%(n=120 / 185)$ of patients, of whom $52 \%(n=62 / 120)$ required mechanical ventilation and $48 \%(n=58 / 120)$ nCPAP. Inotropic support was given to $41 \%$ $(n=75 / 185)$ of children and $27 \%(n=50 / 185)$ received blood product transfusions. Thirtyfive $(70 \%)$ received a blood transfusion, $5(10 \%)$ received fresh frozen plasma and 1 (2\%) received an albumin transfusion. Eight (16\%) of those transfused received both blood and fresh frozen plasma, while 1 received blood and albumin.

The majority (70\%) of patients were successfully discharged, while $24 \%(n=44 / 185)$ were transferred to a dedicated PICU at a central/tertiary hospital and 6\% $(n=12 / 185)$ died (Table 1). All deaths occurred in infants $<1$ year old (median 2.5 months, range newborn to 9.3 months) (Table 1 ).

\section{Premature infants}

Nearly half $(49 \%, n=20 / 41)$ of all neonatal admissions were premature babies $(<37$ completed weeks' gestation) with a median gestational age of 29 weeks (range 29 36 weeks) and a median age on admission of 3 days (range 0 - 27 days). The male to female ratio was 2.3:1. The median duration of stay was 1.82 days (range 4 hours - 13.4 days) and $65 \%$ were admitted after hours. Forty per cent of premature infants had low birth weight, $45 \%$ very low birth weight and $10 \%$ had an extremely low birth weight. Sepsis (45\%, $n=9 / 20$ patients) was the main reason for admission, followed by hyaline membrane disease $(30 \%, n=6 / 20)$ and apnoea $(25 \%$, $n=5 / 20)$. Fourteen $(70 \%)$ premature infants were successfully discharged, 4 (20\%) were transferred to a central hospital and 2 babies (10\%) died. The 2 deaths that occurred were due to sepsis and pneumonia.

\section{Term infants}

Of the neonates admitted, $51 \%$ were fullterm with a mean age of 7 days (range newborn - 25 days). Seven of these neonates (33\%, $n=7 / 21)$ were small for gestational age, the rest being normal for gestational age. A

Table 1. Outcome of neonates and children admitted to a general high-care unit

\begin{tabular}{llll}
\hline Age group & Step down/discharge & Referral to tertiary hospital & Death \\
\hline$\leq 28$ days & 27 & 10 & 4 \\
29 days - 6 months & 55 & 14 & 7 \\
$>6$ months - 1 year & 28 & 8 & 1 \\
$>$ 1 year - 5 years & 16 & 11 & 0 \\
$>5$ years & 3 & 1 & 0 \\
Total & 129 & 44 & 12 \\
$\%$ of total admissions & 70 & 24 & 6
\end{tabular}

Table 2. Age ranges, nutritional assessment and outcomes of patients admitted to the general high-care unit

\begin{tabular}{|c|c|c|c|c|}
\hline Data & $\begin{array}{l}29 \text { days - } 6 \text { months } \\
(n=76)\end{array}$ & $\begin{array}{l}>6 \text { months - } 1 \text { year } \\
(n=37)\end{array}$ & $\begin{array}{l}>1-5 \text { years } \\
(n=27)\end{array}$ & $\begin{array}{l}>5 \text { years } \\
(n=4)\end{array}$ \\
\hline Age (mo), mean & 3.1 & 8.52 & 22.64 & 99.86 \\
\hline Male:female ratio & $1.71: 1$ & $1.05: 1$ & $1.25: 1$ & $1: 3$ \\
\hline $\begin{array}{l}\text { Duration of stay } \\
\text { (days), mean }\end{array}$ & 2.46 & 2.35 & 1.75 & 2.47 \\
\hline WAZ, mean & -2.13 & -1.94 & -1.76 & -1.79 \\
\hline $\begin{array}{l}\text { Admissions after } \\
\text { hours, } n(\%)\end{array}$ & $48(63)$ & $22(59)$ & $15(56)$ & $4(100)$ \\
\hline Death, $n(\%)$ & $7(9)$ & $1(3)$ & 0 & 0 \\
\hline Transfer out, $n(\%)$ & $14(18)$ & $8(22)$ & $8(30)$ & $1(25)$ \\
\hline $\begin{array}{l}\text { Successful } \\
\text { discharge, } n(\%)\end{array}$ & $55(72)$ & $28(76)$ & $16(59)$ & $3(75)$ \\
\hline
\end{tabular}

total of $61 \%$ of the admissions occurred after hours. The median duration of high-care stay was 1.7 days (range 1.5 hours - 12.3 days). The majority of patiets $(62 \%, n=13 / 20)$ were successfully discharged, while $29 \%(n=6 / 21)$ were transferred to a central hospital and 2 (9\%) died. AGE $(38 \%, n=8 / 21)$ was the most common cause of admission for full-term babies, followed by sepsis (19\%, $n=4 / 21$ patients) and birth asphyxia (19\%, $n=4 / 21$ patients). In both neonates who died, the cause of death was birth asphyxia, complicated by pulmonary hypertension.

\section{Older children}

The vast majority $(77 \%, n=142 / 185)$ of all admissions were children between the ages of 1 month and 1 year; 8 out of the 12 deaths occurred in this age group (Table 2). The median age of this group was 5.6 months (range 1 month - 12.5 years). The majority (62\%) were admitted after hours. Most (73\%) of these infants were successfully discharged, $20 \%$ were transferred to a tertiary facility, and $6 \%$ died.

The most common diagnoses for children older than 1 month of age were ALRTI $(42 \%, n=61 / 144)$ and AGE $(36 \%, n=52 / 144)$ (Fig. 2). In this group, $84 \%$ required ventilator support, with $77 \%$ requiring nCPAP and $23 \%$ mechanical ventilation. In the children with ALRTI, there were 4/61 deaths (7\%) and $14 / 61$ were (23\%) transferred to a PICU. In addition to these 4 deaths, there were 4 additional deaths, 3 from complicated gastroenteritis. Of these deaths, $85 \%$ were admitted after hours.

Children older than 1 year of age accounted for $17 \%(39 / 185)$ of the total admissions, with only 4 children admitted being older than 5 years of age. Of those younger than 5 years, the median age was 15.6 months. Table 2 shows outcomes and nutritional status. No children older than 1 year of age died.

The HIV status of $37 \%(n=69)$ of patients was unknown at the time of admission. Five children were known to be HIV-infected on admission and $15 \%$ were known to be HIV-exposed. The remainder $(n=83,45 \%)$ were unexposed and known to be HIVnegative at the time of admission. The majority of patients older than 1 month of age $(n=112 / 129)$ were tested for HIV. DNA polymerase chain reaction was performed for 11 patients, and 5 patients were newly diagnosed with HIV. One of the known HIV-infected patients received antiretroviral 
Table 3. Comparing deaths, transfers and successful discharges of neonates and children admitted to a general high-care unit

\begin{tabular}{|c|c|c|c|}
\hline & Deaths $(n=12)$ & Transfers $(n=44)$ & $\begin{array}{l}\text { Successful } \\
\text { discharges }(n=119)\end{array}$ \\
\hline Male:female ratio & $2: 1$ & $1: 1$ & $1.4: 1$ \\
\hline \multicolumn{4}{|l|}{ Age (mo) } \\
\hline Mean & 2.6 & 11.9 & 7.8 \\
\hline Median & 2.4 & 4.06 & 3.9 \\
\hline Range & $0-9.2$ & $0-12$ & $0-9.5$ \\
\hline Admissions after hours (\%) & 83 & 45 & 64 \\
\hline \multicolumn{4}{|l|}{ Duration of stay (days) } \\
\hline Mean & 1.6 & 1.5 & 2.9 \\
\hline Median & 1.01 & 1.2 & 2.0 \\
\hline Range & $0.04-5$ & $0.08-5.1$ & $0.04-13.4$ \\
\hline \multicolumn{4}{|l|}{ WAZ } \\
\hline Mean & $-1.60 \mathrm{SD}$ & $-1.40 \mathrm{SD}$ & $-1.91 \mathrm{SD}$ \\
\hline Median & $-1.30 \mathrm{SD}$ & $-1.49 \mathrm{SD}$ & $-1.63 \mathrm{SD}$ \\
\hline Range & $-4.80-0.89 \mathrm{SD}$ & $-5.20-1.88 \mathrm{SD}$ & $-6.05-1.57 \mathrm{SD}$ \\
\hline \multicolumn{4}{|l|}{ Interventions (\%) } \\
\hline 0 & 8 & 7 & 18 \\
\hline 1 & 17 & 48 & 52 \\
\hline 2 & 50 & 32 & 22 \\
\hline 3 & 25 & 13 & 8 \\
\hline ALRTI (\%) & 33 & 32 & 35 \\
\hline AGE (\%) & 25 & 23 & 37 \\
\hline
\end{tabular}

(ARV) therapy at the time of admission. None of the newly diagnosed HIV-positive children were started on ARV therapy during their admission to the unit. Six of the HIV-positive patients $(60 \%, n=6 / 10)$ were successfully discharged, $3(30 \%, n=3 / 10)$ were transferred and 1 child died (10\%, $n=1 / 10)$.

The majority $(62 \%, 32 / 52)$ of patients admitted with AGE were admitted due to hypovolaemic shock not responding to fluid resuscitation. Of these children, $85 \%$ required inotropic support. AGE was complicated by severe malnutrition in $13 \%$ $(n=7 / 52)$ of cases, but all these children were successfully discharged despite their poor nutritional states. Three patients admitted with AGE died $(n=3 / 52,6 \%)$ andeight patients $(n=8 / 52,15 \%)$ were transferred to a tertiary facility.

Five patients were admitted with a diagnosis of $\mathrm{TB}$, of whom four had comorbid disease (respectively HIV, dilated cardiomyopathy, lower airway obstruction and TB meningitis). One child with complicated pulmonary TB had no complicating comorbid disease. Three of these patients were transferred and two were successfully discharged. Five patients were admitted due to upper airways obstruction (UAO) and the majority $(60 \%$, $3 / 5)$ required mechanical ventilation and transfer. Two patients were successfully discharged. There were two patients admitted with acute flaccid paralysis (AFP), of whom one was successfully discharged; the second patient required long-term mechanical ventilation and was transferred. Two patients had acute liver failure due to ingestion of unknown herbal medication. Both of these children were transferred (Table 3 ).

Of the patients who died, $75 \%$ required two or more interventions, compared with $45 \%$ of patients transferred to a dedicated PICU and $30 \%$ of patients successfully discharged. Patients requiring nCPAP or mechanical ventilation were more likely to die or require transfer $(p=0.0001)$. A third of the patients received nCPAP, which was associated with either transfer or death $(p<0.001)$. Patients who were diagnosed with HIV infection or patients known to be HIV-positive, had a statistically significant poorer outcome when compared with HIV non-infected children $(p=0.01)$ (Table 3$)$. Only $28 \%$ of patients received a transfusion of one or more blood products, but neither the use of blood products ( $p=0.87)$, nor the need for inotropic support ( $p=0.16$ ) was significantly associated with transfer or death. Although AGE and ALRTI were responsible for more than 50\% of admissions, there was no statistically significant association between diagnosis and outcome $(p=0.17)$. Complications of prematurity (sepsis, hyaline membrane disease and apnoea) and neonates referred from outside healthcare facilities were not associated with poor outcome $(p=0.98$ and $p=0.38$, respectively). There was a trend towards a significant association $(p=0.07)$ between time of admission and outcome in this study, with after-hour admissions tending to have a poorer outcome. The prevalence of severe malnutrition among admissions in this study was $25 \%$, but there was no association between nutritional status and a poor outcome $(p=0.56)$. Although the majority of the patients were younger than 12 months (median age 3.7 months), age was not associated with a poor outcome $(p=0.17)$. In summary, the majority of children requiring access to high-care unit in a regional hospital, were younger than 1 year of age and $70 \%$ had a good outcome. The need for good ventilatory support and being HIV-infected was associated with a poor outcome.

\section{Discussion}

In this study we describe the good outcome achieved in children admitted to a general high-care in a regional hospital in the Western Cape. The reported low mortality rate of $6.5 \%$ was not solely due to the care in the general high-care unit but also, in large part, due to the fact that there was an effective emergency transport system which allowed for $24 \%$ of the children to be transported to a PICU within $100 \mathrm{~km}$ of the regional hospital. The study also identified mechanical ventilation and HIV infection as risk factors for transfer to a PICU, which would in future allow for a change in transfer policy resulting in children being transferred earlier for PICU care. The study only included children not requiring surgery. Children with serious trauma and burn wounds were not admitted to the high-care unit but were directly transferred, after resuscitation, to the appropriate tertiary hospital. This policy together with the fact that only minor surgical procedures were performed would have contributed to the low mortality rate.

Age has been reported to be associated with a poor outcome within the PICU. ${ }^{[6]}$ In this study we were not able to demonstrate that age was statistically associated with death or transfer. It should however be noted that $8(75 \%)$ of the deaths occurred in children less than 1 year of age. The two most common causes of death in children $<1$ year of age were complicated ALRTI and AGE, both potentially treatable conditions. It was noted that $85 \%$ of the children older 
than 28 days of age who died were admitted after hours. This might suggest that caregivers did not have easy access to after-hours healthcare facilities, that the emergency transport services did not operate as efficiently after hours, that the healthcare after hours in the regional hospitals is mainly run by junior staff who might underestimate the acuity of disease, or that it is a combination of these reasons. Further research is required to elicit the reason for the poor outcome after hours.

ALRTI, AGE, birth asphyxia and complications of prematurity are reported as the main causes of death in children $<5$ years of age. This was confirmed in this study, where ALRTI was the leading cause of both admissions and deaths, followed by AGE. ${ }^{[2,11]}$ It is estimated that $20 \%$ of infants with birth asphyxia will die within the neonatal period. ${ }^{[12]}$ Only 4 term infants were admitted with birth asphyxia in this study and 2 died. A study limitation is the small sample size and death rate (per 1000 ) due to birth asphyxia, which could not be established. Severe malnutrition is reported in $18-65 \%$ of all paediatric admissions and 15 - 65\% of admissions to PICU and has been linked to poor outcome, reporting mortality rates up to $30 \%{ }^{[13]}$ However, differing methodologies used to classify malnutrition make it difficult to compare the incidence and mortality rates between different studies. ${ }^{[13]}$ In this study one of the weaknesses was establishing the exact weight of the neonates and children admitted. This might have resulted in underestimation of the weights of the children, especially those admitted with severe dehydration. Even taking this into account, $25 \%$ of the children were considered to be severely malnourished, indicating that their complicated disease was multifactorial. Invasive ventilation has been reported to be associated with a poorer outcome in PICUs, which was similar to our findings $(p<0.001) \cdot{ }^{[14]}$ nCPAP has become a common intervention to provide airway support for ALRTI, with up to $10 \%$ of children admitted to paediatric intensive care receiving non-invasive airway support. ${ }^{[15]}$ The addition of non-invasive airway support (including nCPAP) is associated with improved outcome without contributing significantly to adverse events. ${ }^{[14]}$ In this study, children receiving nCPAP had an increased rate of transfer to a PICU and death. The use of nCPAP and mechanical ventilation indicates that these children had severe ALRTI resulting in respiratory failure with a high proportion of children requiring transfer to a PICU. This finding illustrates the point that a general high-care unit cannot operate in isolation but requires good emergency transport services and access to PICU beds in a tertiary hospital that is within easy reach. If this were not the case it is postulated that especially the infant mortality rate would have been unacceptably high. On the other hand, $62(33.5 \%)$ neonates and children required ventilation which would not have been possible if they were not admitted to the high-care unit. This illustrates the importance of having these facilities available in regional hospitals as a minimum to stabilise the neonates and children prior to transfer to a central intensive care unit. The addition of inotropic support to the treatment of patients in our study was not significantly associated with a poorer outcome, which also differs from other studies, where the addition of inotropes to treatment was a risk factor for death during intensive care unit stay. ${ }^{[16]}$ Transfusion of blood products is commonly used (up to $50 \%$ of admissions) in critically ill children admitted to PICUs, whereas only $28 \%$ of patients in this study received blood product transfusions and there was no association with poor outcome. ${ }^{[17]}$

Limitations of the study include a short study period, small sample size and no objective measurement of the patients' disease acuity. Another weakness to take into account is that the outcomes of the patients transferred to a PICU were not determined. This is further compounded by the fact that critically ill patients were also directly transferred from surrounding healthcare facilities to the regional hospital. The outcome of patients referred to tertiary care is not discussed as part of this paper and the reasons for refusal of admission to the unit or to the tertiary referral centre are not included. The data in this study are relatively old, but to our knowledge a study of this kind has not been reported in the interim, illustrating the value of having access to a high-care unit in a regional hospital in SA. A prospective study with routine collection of more comprehensive data, including causes of morbidity and mortality linked to ICD-10 codes, assessments of disease severity and interventions, needs to be performed to provide comprehensive information on the value of admitting neonates and children to a high-care or critical-care unit in a regional hospital. To our knowledge this is the first study reporting admissions and outcome of neonates and children cared for in a general high-care unit where the care is given by general paediatricians working in a regional hospital in SA. This study suggests that these units in large regional hospitals, with paediatricians supporting their care, may improve child survival in SA if the high care is part of a functional system that includes transport of the severely ill to tertiary facilities with paediatric intensive care facilities. More data are required to support this hypothesis.

\section{References}

1. Black RE, Morris SS, Bryce J. Where and why are 10 million children dying every year? Lancet 2003;361(9376):2226-2234. DOI:10.1016/S0140-6736(03)13779-8

2. Jones G, Steketee RW, Black RE, et al. How many child deaths can we prevent this year? Lancet 2003;362(9377):65-71. DOI:10.1016/S0140-6736(03)13811-1

3. Baker T. Critical care in low-income countries. Trop Med Int Health 2009;14(2):143-148. DOI:10.1111/j.1365-3156.2008.02202.x

4. Baker T. Pediatric emergency and critical care in low-income countries. Paediatr Anaesth 2009;19(1):23-27. DOI:10.1111/j.1460-9592.2008.02868.x

5. Argent A. Critical care in Africa. S Afr J Crit Care 2009;25(1):4.

6. Basnet S, Adhikari N, Koirala J. Challenges in setting up pediatric and neonatal intensive care units in a resource-limited country. Pediatrics 2011;128(4):e986-e992. DOI:10.1542/peds.2010-3657

7. Randolph AG, Gonzales CA, Cortellini L, Yeh TS. Growth of pediatric intensive care units in the United States from 1995 to 2001. J Pediatr 2004;144(6):792798. DOI:10.1016/j.jpeds.2004.03.019

8. Campos-Miño S1, Sasbón JS, von Dessauer B. Pediatric intensive care in Latin America. Med Intensiva 2012;36(1):3-10. DOI:10.1016/j.medin.2011.07.004

9. Hatherill M, Waggie Z, Reynolds L, Argent A. Transport of critically ill children in a resource-limited setting. Intensive Care Med 2003;29(9):1547-1554. DOI:10.1007/s00134-003-1888-7

10. Groenewald P, Berteler M, Bradshaw D, et al. Western Cape Mortality Profile 2010. Cape Town: South African Medical Research Council, 2013.

11. Fenton TR, Nasser R, Eliaziw M, et al. Validating the weight gain of premature infants between the reference growth curve of fetus and term infants. BMC Pediatr 2013;13:59. DOI:10.1186/1471-2431-13-92

12. Wells, M, Riera-Fanego JF, Luyt DK, Dance M, Lipman J. Poor discriminatory performance of the Pediatric Risk of Mortality (PRISM) score in a South African intensive care unit. Crit Care Med 1996;24(9):1507-1513.

13. Jeena PM, McNally LM, Stobie M, Coovadia HM, Adhikari MA, Petros AJ. Challenges in the provision of ICU services to HIV infected children in resource poor settings: A South African case study. J Med Ethics 2005;31(4):226-230. DOI:10.1136/jme.2003.004010

14. Tan GH, Tan TH, Goh DY, Yap HK. Risk factors for predicting mortality in a paediatric intensive care unit. Ann Acad Med Singapore 1998;27(6):813-818.

15. Inwald DP, Tasker RC, Peters MJ, Nadel S, Paediatric Intensive Care Society Study Group (PICS-SG). Emergency management of children with severe sepsis in the United Kingdom: The results of the Paediatric Intensive Care Society sepsis audit. Arch Dis Child 2009;94(5):348-353. DOI:10.1136/ adc.2008.153064

16. Ten Berge J, de Gast-Bakker DA, Plötz FB. Circumstances surrounding dying in the paediatric intensive care unit. BMC Pediatr 2006;6:22. DOI:10.1186/1471-2431-6-22

17. Gauvin F, Spinella PC, Lacroix J, et al. Association between length of storage of transfused red blood cells and multiple organ dysfunction syndrome in pediatric intensive care patients. Transfusion 2010;50(9):1902-1913. DOI:10.1111/j.1537-2995.2010.02661.x

Accepted 7 October 2015 